ISSN 1112-9867

Available online at

http://www.jfas.info

\title{
STRUCTURAL STABILITY AND ELECTRONIC STRUCTURE OF YCU DUCTILE INTERMETALLIC COMPOUND BY FIRST-PRINCIPAL CALCULATION.
}

\author{
A. Sekkal ${ }^{1 *}$ and A. Benzair ${ }^{2}$ \\ ${ }^{1}$ Laboratoire d'Etude et Prédiction de Matériaux, Unité de Recherche Matériaux et Energies \\ Renouvelables, Département de Physique, faculté des Sciences, Université Abou Bekr \\ Belkaid, B.P 119, 13000 Tlemcen, Algérie. \\ ${ }^{2}$ Département de Physique, faculté des sciences, Université Djillali Liabes. 22000 Sidi Bel \\ Abbés, Algérie
}

Received: 29 April 2012 / Accepted: 17 December 2010 / Published online: 31 December 2012

\begin{abstract}
We investigate the structural, elastic and electronic properties of cubic $\mathrm{YCu}$ intermetallic compound. Which crystallize in the $\mathrm{CsCl}-\mathrm{B} 2$ type structure, the investigated using the first principle full potential linearized augmented plane wave method (FP-LAPW) within density functional Theory (DFT). We used generalized gradient approximation (GGA) and local density theory (LDA), which is based on exchange correlation energy optimization, are used to determinate the total energies and the electronic band structures. The latter provide us with the ground states properties such as lattice parameter, bulk modulus and its pressure derivative, elastic constants and the structural phase stability with respect to the $\mathrm{B}_{1}, \mathrm{~B}_{3}$, and $\mathrm{L}_{10}$ structures of this compound. Our results are in good agreement with numerous experimental and theoretical data where available.
\end{abstract}

Key words: Rare-Earth Alloy, phase stability, Density of state, elastic constants, ab initio calculations.

\section{INTRODUCTION}

The inter-metallic compounds typically possess Chemical, physical, and mechanical properties superior to ordinary metals. However, their room temperature brittleness and poor fracture toughness severely restrict their use.

Author Correspondence, e-mail: asamad2002@yahoo.fr

ICID: 1025915 
The study of the inter-metallic compounds such as $\mathrm{YCu}$ has attracted the attention of the scientific world because of their anisotropic properties [1], it has also ductile. While there may be applications at high temperature strength and corrosion resistance. This alloy has the B2 structure, like that found in cesium-chloride $(\mathrm{CsCl})$ and chemical formula $\mathrm{RM}$, where $\mathrm{R}$ denotes a rare - earth element and $\mathrm{M}$ denotes a late transition metal or an early p-element.

Only a few theoretical papers and little experimental work have been devoted to the study of structural and electronic properties of this binary compound. The electronic structure, density of states and crystal field in rare earth inter-metallic were studied for $\mathrm{YCu}$, with the $\mathrm{CsCl}$ structure by Divis and Kuriplach [2] and the crystal field parameters of this compound was calculated by Schmitt [3] and later by Newmann [4]. The rare earth ions in inter-metallic compounds are generally trivalent [5].

In this paper, we present ab-initio calculations of the properties of the $\mathrm{RM}$ compound $\mathrm{YCu}$, including the lattice constants, elastic constants, band structure, density of state and phase stability with the $\mathrm{B} 1, \mathrm{~B} 3$, and $\mathrm{L} 1_{0}$ structure, and find good agreement with experimental values where available.

The rest of the paper is organized as follows. In Section 2, we describe briefly the computational techniques used in this work. Results and discussion of the structural, electronic and elastic properties will be presented in Section 3, and a summary of the work will be given in Section 4 .

\section{CALCULATION METHOD}

The intermetallic compound $\mathrm{YCu}$, which are taken up for investigation crystallize in the cesium chloride structure (B2 type) with the space group symmetry Pm3m [2.6]. Y atoms are positioned at $(0,0,0)$ and $\mathrm{Cu}$ atoms occupy $(1 / 2,1 / 2,1 / 2)$ positions and the lattice parameters are taken from the experimental work [6].The calculations were performed by FP-LAPW method to solve the Kohn Sham equations as implemented in the WIEN2k code [7]. The exchange correlation energy is described in the generalized gradient approximation (GGA) using the Perdew-Wang functional [8] and local density theory (LDA). In this approach the wave function charge density and potential are expanded differently in two regions of the unit cell. Inside the non-overlapping spheres of radius RMT around each atom, spherical harmonics expansion is used and in the remaining space of the unit cell the plane wave basis set is chosen. $\mathrm{RMT}$ values for $\mathrm{Y}$ and $\mathrm{Cu}$ in $\mathrm{CsCl}$ structure are assumed to be 2.0 and 1.87 a.u. We included angular momentum components up to $1=10$, and used 100 or more plane waves 
per atom. We used the energy that separates the valence states from the core states was chosen to be 6.0 Ryd. This is a typical value used for such calculations, The plane wave cutoff of $\mathrm{K}_{\max }=7.0 / \mathrm{R}_{\mathrm{MT}}$ is chosen for the expansion of the wave functions in the interstitial region while the charge density was Fourier expanded up to Gmax $=14.0$. A mesh of 24 special k-points for $\mathrm{YCu}$ structure was taken in the irreducible wedge of the Brillouin zone. Both the MT radius and the number of $\mathrm{k}$ - points were varied to ensure total energy convergence. The following basic orbital is used.

Y: $5 \mathrm{~s}^{2}, 5 \mathrm{p}^{0}, 4 \mathrm{~d}^{1}$

$\mathrm{Cu}: 4 \mathrm{~s}^{1}, 3 \mathrm{~d}^{10}, 4 \mathrm{p}^{0}$

\section{RESULTS AND DISCUSSION}

Using the full-potential LAPW formalism, we have calculated the equilibrium lattice constant of $\mathrm{YCu}$ within the GGA and LDA approximations. In Fig. 1 we show the plots of our calculated total energy as a function of volume for this compound in B1, B2, B3, and L10 phases, as well as the energy curves produced from the fit model equation of state proposed by Murnaghan [9]. It is clear that B2 is the stable structure which is consistent with experimental results and other theoretical works. The calculated equilibrium lattice constants a , bulk modulus B and its pressure derivative B' for structures in B1, B2, B3, and $\mathrm{L}_{10}$ are listed in Table 1 which also contains results of the previous first principle calculations as well as the experimental data.

We calculated lattice constants and bulk modulus are compared to the corresponding experimental and other theoretical results. In general, our calculated lattice constants and bulk modulus are in agreement with results of previous

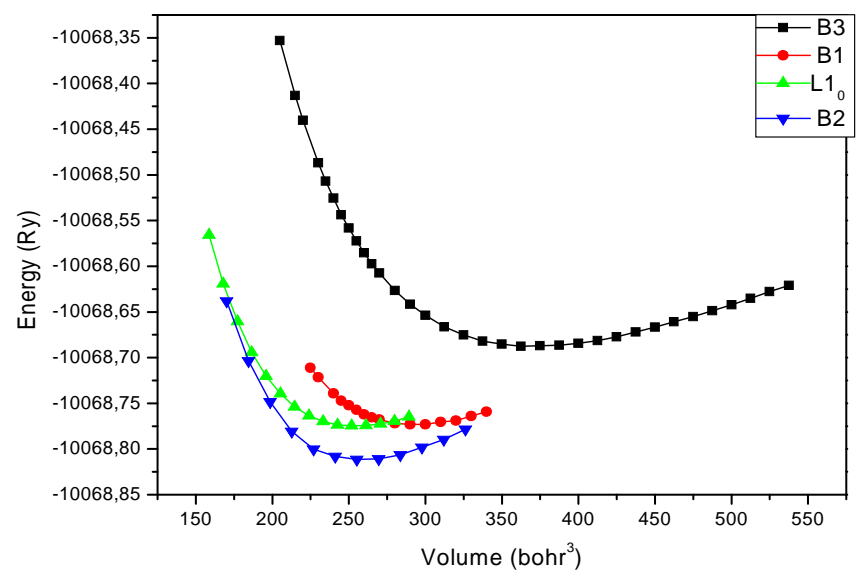

Fig.1. Calculated total energies as a function of volume in the $\mathrm{B} 1, \mathrm{~B} 2, \mathrm{~B} 3$, and $\mathrm{L}_{0}$ structure for $\mathrm{YCu}$ 
studies and experiment. The difference between our calculated structural parameters $\left(\mathrm{a}_{0}, \mathrm{~B}\right)$ and those find by James R. Morris and co-works [10] (for YCu) and Sk. Khadeer Pasha [11] is essentially due to the fact that they used the LDA approximation. In addition, we have calculated the $\mathrm{C}$ ij of these compounds in $\mathrm{CsCl}-\mathrm{B} 2$ phase at zero temperature and zero under hydrostatic pressure B2 structure at the equilibrium volumes using the method discussed in detail in Ref. [12]. In our calculations, we have considered only small lattice distortion in order to remain within elastic domain of the crystal. A cubic structure is characterized by three independent elastic constants namely $\mathrm{C}_{11}, \mathrm{C}_{12}$, and $\mathrm{C}_{44}$. The calculated values of the elastic constants $\mathrm{C}_{\mathrm{ij}}$ are listed in Table 1. In all cases the comparison is quite agreeable. The largest discrepancy between theory and experiment is the value for $\mathrm{C}_{12}$, where the difference is approximately $4.24 \%$. The value for $\mathrm{C}_{11}$ is also different by $3 \%$. It is noticeable that for this compounds, all elastic constants satisfies the following criterions: $\mathrm{C}_{11}>0 ; \mathrm{C}_{44}>0 ; \mathrm{C}_{11}+2 \mathrm{C}_{12}>0 ; \mathrm{C}_{11}-\mathrm{C}_{12}>0$. Also, the bulk modulus also satisfy a criterion $\mathrm{C}_{12}$ $<\mathrm{B}<\mathrm{C}_{11}$ implying that those compounds are stable with elastic deformation.

\begin{tabular}{|c|c|c|c|c|c|}
\hline \multirow[t]{2}{*}{ Phase } & & \multicolumn{2}{|c|}{ Present work } & \multirow[t]{2}{*}{ Experiment } & \multirow{2}{*}{$\begin{array}{l}\text { Other theoretical } \\
\text { works }\end{array}$} \\
\hline & & LDA & GGA & & \\
\hline \multirow{6}{*}{ B2 } & $a_{0}\left(A^{0}\right)$ & 3.373 & 3.478 & 3.477 & $3.4770^{\mathrm{a}}, 3.424^{\mathrm{b}}$ \\
\hline & B & 84.925 & 70.149 & 70.1 & $70^{\mathrm{a}}, 97^{\mathrm{b}}$ \\
\hline & B' & 3.657 & 3.585 & - & - \\
\hline & $\mathrm{C}_{11}$ & 114.833 & 116.949 & 113.4 & $116^{\mathrm{a}}$ \\
\hline & $\mathrm{C}_{12}$ & 68.270 & 46.432 & 48.4 & $47^{\mathrm{a}}$ \\
\hline & $\mathrm{C}_{44}$ & 32.050 & 50.176 & 32.3 & $35^{\mathrm{a}}$ \\
\hline \multirow[b]{3}{*}{ B1 } & $\mathrm{a}_{0}\left(\mathrm{~A}^{0}\right)$ & 5.591 & - & - & - \\
\hline & B & 72.157 & - & - & - \\
\hline & $\mathrm{B}^{\prime}$ & 4.012 & - & - & - \\
\hline \multirow{3}{*}{ B3 } & $\mathrm{a}_{0}\left(\mathrm{~A}^{0}\right)$ & 6.063 & - & - & - \\
\hline & B & 48.007 & - & - & - \\
\hline & $\mathrm{B}^{\prime}$ & 3.557 & - & - & - \\
\hline \multirow{4}{*}{ L10 } & $a_{0}\left(A^{0}\right)$ & 4.231 & - & - & - \\
\hline & $c\left(A^{0}\right)$ & 4.823 & - & - & - \\
\hline & $\mathrm{B}$ & 82.274 & - & - & - \\
\hline & $\mathrm{B}^{\prime}$ & 3.41 & - & - & - \\
\hline
\end{tabular}

(a):FPLAPW,ref[10].(b):TBLMTO-ASA, ref[11]

Table 1. The calculated lattice constant $\mathrm{a}_{0}$ (in $\mathrm{A}^{\circ}$ ), bulk modulus $\mathrm{B}$ (in GPa), pressure derivative (B') of $\mathrm{YCu}$ for B1, B2, B3, and $\mathrm{L} 10$ phase, and elastic constants $\mathrm{Cij}$ (in GPa) for B2 phase.

We calculated band structure of $\mathrm{YCu}$ compound in the $\mathrm{B}_{2}$ phase at equilibrium volume using the FP-LAPW method within DFT along the higher symmetry directions is presented in Fig. 2. The band structure of this phase show large dispersion of rather free electron type which 
indicate metallic character, in this Band, the lowest lying band is mainly due to $\mathrm{Cu}$ "d" like electrons. The bands above this and below the Fermi energy arise due to Y "s" like states. The states just above the Fermi level are due to $\mathrm{Y}$ " $\mathrm{d}$ " like electrons. In the present work are in good agreement with the work of Sk. Khadeer Pasha [11].The total DOS has also been plotted for $\mathrm{YCu}$ compound and is shown in Fig.3.This figure shown that the large occupied peak is dominated by the $\mathrm{Cu}$ " $\mathrm{d}$ " state and the lattice is distorted so as to position the Fermi level at a local minimum in the DOS. This creates a local gain in the electronic contribution to the energy, the Fermi energy lies near a local minimum, and the bonding states are only partially full. Thus, the bonding is relatively insensitive to local distortions. The partial densities of states shown in Fig. 4, shows that in $\mathrm{YCu}$, the density of d-states at the Fermi energy is low.

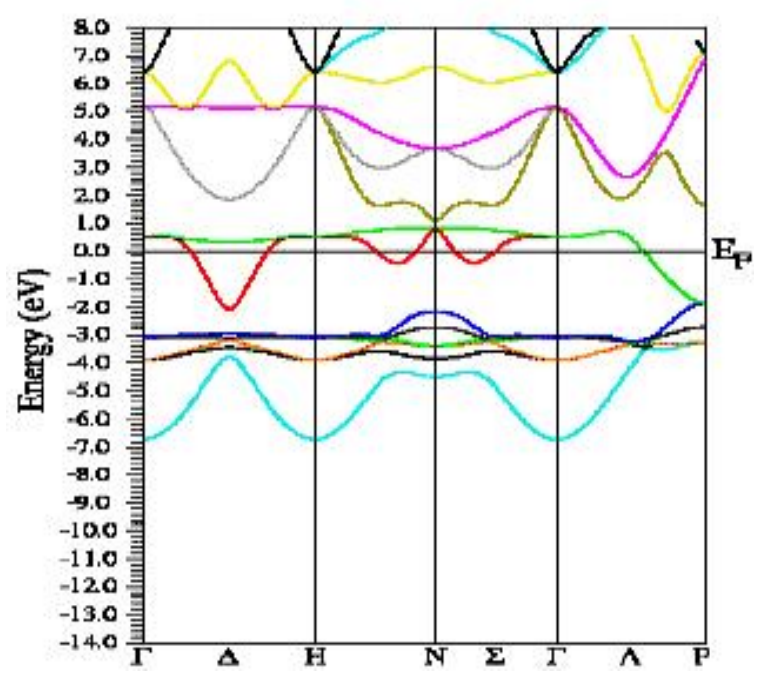

Fig.2. Band structure in the B2 phase for $\mathrm{YCu}$

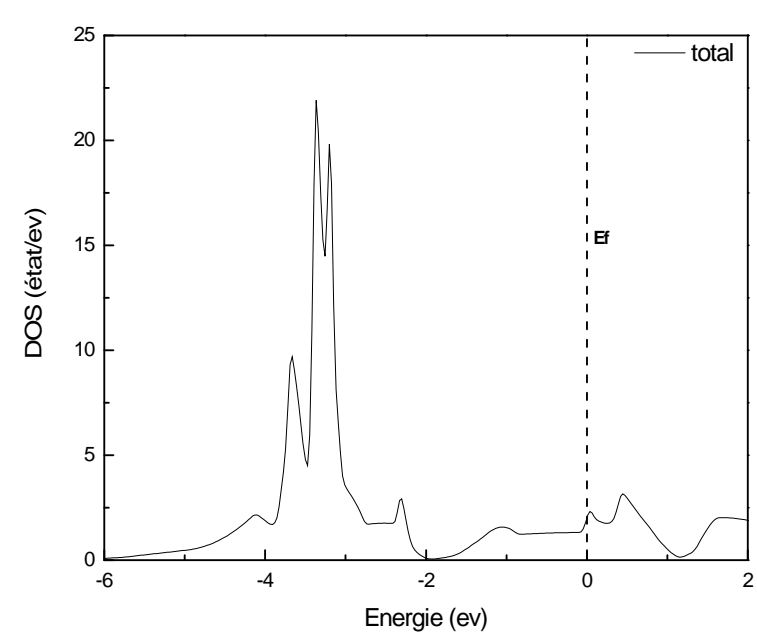

Fig.3. Total electronic density of states (DOS)

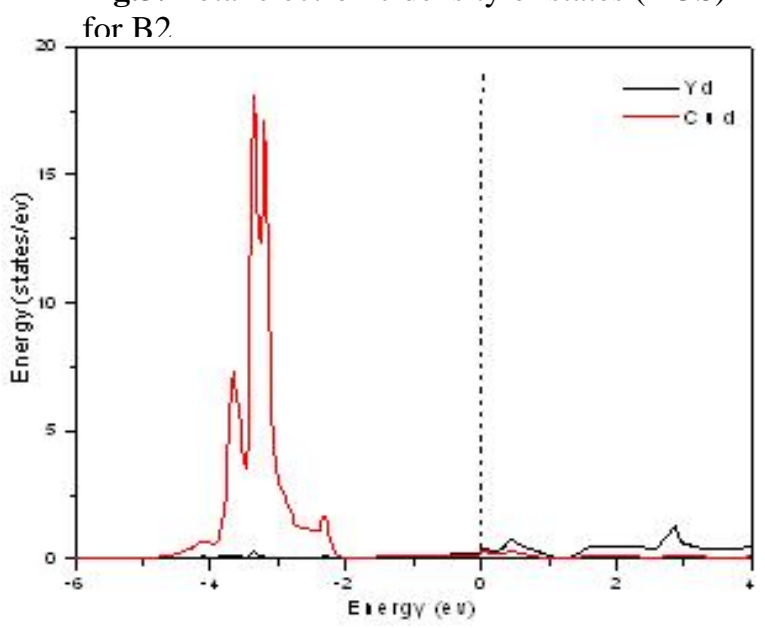

Fig.4. Partial density of states for $\mathrm{YCu}$, showing the $\mathrm{Y}$ and $\mathrm{Cu}$ 'd'-states 
In comparison between the GGA and LDA approximation, we conclude that LDA underestimate the lattice parameter and the bulk modulus of $\mathrm{YCu}$ in $\mathrm{B} 2(\mathrm{CsCl})$ phase.

In other hand, we find a good agreement between our result of the electronic band structure and the other theoretical works.

\section{CONCLUSION}

In the present study, we have presented a complete theoretical analysis of the structural, elastic and electronic properties of the inter-metallic compound, namely $\mathrm{YCu}$ by using the FPLAPW method. The use of GGA and LDA for the exchange-correlation potential permitted us to obtain good structural parameters. Our result indicate that B2 is more stable than B1, B2, and L10.we find that the lattice constant, The Bulk modulus, and constants elastic $\left(\mathrm{C}_{11}, \mathrm{C}_{12}\right.$ and $\mathrm{C}_{44}$ ) are in excellent agreement with available experimental data and other calculations.

\section{REFERENCES}

[1] Divis M., Kuriplach J., Physica 1993, B 183, 24

[2] Divis M., Kuriplach J. Physica 1995, B 205, 353.

[3] D. Schmitt, J. Phys. 1979, F 9, 1745.

[4] Newmann D.J. J. Phys. 1983, F 13,1511.

[5] Fulde P., Loewenhaupt M. Adv.Phys. 1986, 34, 589.

[6] Buschow K.H.J., Rep. Prog. Phys. 1979, 42, 1373.

[7] Blaha P., Schwarz K., Madsen G.K.H., Kvasnicka D. J. Luitz WIEN2k, An Augmented Plane Wave Plus Local Orbitals Program for Calculating Crystal Properties, Vienna University of Technology, Vienna, Austria, 2001.

[8] Perdew JP, Wang Y. Phys Rev. 1992, B, 45:13244.

[9] Murnaghan F.D., Proc. Natl. Acad. Sci. USA. 1944, 30, 244.

[10] James R. Morris, Yiying Ye, Yong-Bin Lee, Bruce N. Harmon, Karl A. Gschneidner Jr. Alan M. Russell. Acta Materialia. 2004, 52, 4851.

[11] Khadeer Pasha Sk., Sundareswari M., Rajagopalan M. Physica. 2004, B 348, 207.

[12] Mehl M.J. Phys. Rev. 1993, B 47, 2493.

\section{How to cite this article}

Sekkal A and Benzair A. Structural stability and electronic structure of $\mathrm{YCu}$ ductile intermetallic compound by first-principal calculation. J Fundam Appl Sci. 2012, 4(2), 121126. 